\title{
LAS UNIVERSIDADES PÚBLICAS EN LA SOCIEDAD DEL CONOCIMIENTO
}

\author{
Máximo Ugarte Vega Centeno ${ }^{1}$ \\ mugartev@yahoo.com
}

RESUMEN

El debate sobre la universidad sigue vigente, la situación actual es preocupante por muchos factores internos y externos que influyen en su gestión y sus perspectivas de desarrollo hacia el futuro en una sociedad cambiante, en la que la universidad debe estar a la altura de los acontecimientos para responder y cumplir el rol que tiene asignado en la sociedad.

Palabras Clave: Universidad y Conocimiento

\section{ABSTRACT}

The debate about the University is still in force, the current situation is worrying for many internal and external factors that influence its management and its development prospects for the future in a changing society, in which the University must keep up the events to respond and fulfill the role assigned to it in society.

Keywords: University and Knowledge

\footnotetext{
1 Diplomado en Gestión por el Programa de Alta Dirección (PAD), Universidad de Piura; Magister por la Universidad de Barcelona (España) y Doctor en Estudios Internacionales por la Universidad de Córdoba (España). Profesor Principal de la Universidad Nacional Mayor de San Marcos.
} 


\section{INTRODUCCIÓN}

Cuando hablamos de globalización de la economía y la sociedad del conocimiento es preciso vincularlo con las universidades, porque la globalización exigente también llegó a estos centros de estudios; por lo tanto, las universidades y escuelas de post grado tienen y deben estar a la altura de las exigencias.

América Latina está comprometida en este complejo proceso de transformaciones económicas y sociales, en esta aldea global donde los conocimientos están al frente de estos cambios y el sistema universitario tiene que modificar sus organizaciones para responder a los nuevos desafíos.

El sistema universitario en el Perú no está al margen de estos acontecimientos y para insertarse en este escenario competitivo es necesario estar preparados y programar estrategias que nos ayuden a superar estos desafíos, como la capacitación de los recursos humanos, la competitividad en base a la calidad de la enseñanza, la investigación y la actualización de los planes de estudios, acceso al mercado laboral de los egresados y las tecnologías de información y comunicación como socias en este proceso de insertación.

A partir de 1950 se inició una nueva era de la información, la tecnología y del conocimiento. En esa década, en los países desarrollados, muchas personas dejaron de trabajar en la manufactura para pasar a manejar la información, datos estadísticos y conocimientos especializados, servicios, con una visión de liderazgo. En la era de los conocimientos dejaron de usar sus manos y músculos y hoy utilizan las herramientas de la capacitación permanente como una fuente de supervivencia y competitividad para su sobrevivencia y seguridad futura.Como bien sabemos la educación es la base que tiene un país para el desarrollo social, por lo mismo que proporciona conocimientos y destrezas que permiten aumentar la productividad del trabajo y, como consecuencia, los ingresos de las personas.

En ese sentido, algunos plantean que el rol fundamental de la universidad es ordenar el conocimiento existente. La crisis por la que atraviesan todas las universidades del mundo no está vinculada al impacto que el mercado tiene sobre un mayor comportamiento mercantilista.
En otras palabras, no radica en que la universidad esté vendiendo su alma al mercado sino que el problema es más de fondo: es la incapacidad o imposibilidad de cumplir su rol esencial, esto es, construir una visión coherente y compartida de sociedad en permanente cambio.

El siglo XXI se considera el siglo del conocimiento y de la información; luego, resulta bastante paradójico que siendo la universidad la institución clave, tanto en el conocimiento como en la información, esté siendo cuestionada desde diversos flancos. Cuestionando la disminución de los recursos públicos para las universidades, el gasto en educación superior -el cual compite con el gasto en salud y los programas sociales- y los fondos destinados a la educación primaria y secundaria; limitando por tanto dichos recursos para la investigación en ciencia y tecnología.

Hoy la universidad, como organización social, transita hacia nuevos modelos, encaminados a la formación de individuos calificados para el ejercicio profesional, pero también con el compromiso de generar conocimientos útiles para el desarrollo de la sociedad; de ahí la necesidad de articular ciencia, tecnología y gestión del conocimiento en la universidad de principios del siglo XXI.

Ante los desafíos actuales las universidades en el país deben asumir su compromiso social en cualquier perspectiva de desarrollo del país, dotadas de un sistema de gobierno idóneo y con los medios suficientes para investigar con calidad, crear, producir, difundir y gestionar conocimiento tanto científico como tecnológico, adoptando nuevas formas de pensar y de actuar en el cambiante entorno conformado por un mundo cada vez más complejo y desafiante, entonces es posible hacer frente a los retos de la globalización de las economías de mercado ${ }^{2}$.

\section{HIPÓTESIS}

La situación actual y los problemas de la universidad y los postgrados se deben a la influencia de factores externos que influyen en su gestión y en sus perspectivas de desarrollo.

\section{ANÁLISIS Y DISCUSIÓN}

Las particularidades del estudio nos llevan a tener en consideración las siguientes variables de la hipótesis planteada:

2 Stiglitz, Joseph E. (2002). El malestar en la globalización. Madrid, Taurus-Santillana, pp. 308 y 309. 
1. La situación actual de la universidad y los problemas que viene enfrentando;

2. Cuáles serían las perspectivas que tiene o debe tener la universidad y las escuelas de post grado.

1. La situación actual de la universidad y los problemas que viene enfrentando

\subsection{Antecedentes}

Para una mejor comprensión del trabajo de investigación, nos remontaremos, en términos generales, a sus inicios de la educación y la universidad.

El nombre de universidad deriva de la palabra latina "universitas", que significa un establecimiento o conjunto de unidades educativas de enseñanza superior, surgida en la antiguiedad. Adopta este nombre en la edad media europea y se extiende por el mundo gracias a las colonias creadas por diversos países europeos. Las universidades más antiguas podemos encontrarlas en diferentes civilizaciones como en China, donde funcionó la Escuela Superior Imperial ShangHsiang durante el período 22572208 A.C, y la actual universidad de Nankin se remonta a la Academia Central Imperial de Nanking, fundada en el año 258.

En otro ámbito tenemos las universidades persas y árabes que funcionaron durante el siglo IV y finales del V, como las Escuelas de Edesa y Nisbis fundadas por sirios y cristianos nestorianos, y que eran comunidades de maestros dedicadas a la exegesis bíblica3.En el ámbito europeo las primeras universidades son las de Salerno (siglo IX, especializada en medicina), Bolonia (especializada en derecho), y París. Cada una de ellas respondió a exigencias particulares y diferentes, pero todas son igualmente testimonio claro de formas de educación destinadas a repercutir y propagarse por toda Europa.

Hoy tenemos distintos modelos como la universidad inglesa, francesa, española, alemana, norteamericana, latinoamericana, japonesa, china, islámica entre otras.

"Pero en nuestra época la palabra universidad, aplicada al terreno cultural, significa lo que más específicamente se indica en italiano con la expresión "universitádeglistudi" (universidad de los estudios), esto es, la institución cultural o escuela de grado superior que comprende o aspira a comprender la totalidad de las ramas del conocimiento humano, la universalidad de las clases de especialización del saber y de las formas de preparación científica y técnica superior para el ejercicio de las distintas profesiones intelectuales" 4 .

La historia de la educación en el Perú ha estado relacionada con los demás países del mundo; es así que podemos sintetizar su desarrollo en dos fases como lo señala OngaroEstrada. El proceso peruano se ha desarrollado en dos etapas históricas que ha dado horizontes culturales diversos: una primera fase del desarrollo autónomo y de aislamiento geográfico, que dura más de 10,000 años hasta el siglo XI (1532), sujeta a su propia creatividad, configurándose el desarrollo de la civilización Incaica, comparable a las culturas Azteca de México, Maya de Guatemala (en América Central), y las culturas de oriente como Egipto, India, China, etc. Tenemos una segunda fase de dependencia-dominación, que dura cuatro siglos y medio, desde 1532 hasta nuestros días, donde el Perú se ha desenvuelto en la periferia de las sociedades dominantes: España, Inglaterra y Estados Unidos de NorteAmérica5. En ese sentido la historia política de toda sociedad organizada en cada época tuvo su sistema de educación y políticas de educación que respondían, a su vez, a intereses de ese período de su vida económica, social y cultural. Algunos autores6 dividen la historia de la educación en el Perúen la educación del Tahuantinsuyo hasta 1532, La colonia (1532-1821), La Republicana Aristocrática (1821-1920) y la educación actual de 1920 hasta la fecha.

A través de las diferentes constituciones del Perú se ha tratado a la educación superior desde el propósito de fomentarla, considerando la libertad de cátedra, lo que se dio hasta la Carta Magna de 1979, donde se legisla claramente sobre la universidad (artículos 31, 246 y 287), estableciendo sus fines y facultades, asignándole un presupuesto por parte del

\footnotetext{
3 Ver: "Universidad", en www.wikipedia.org/wiki/universidad

4 Al respecto véase Mondolfo, Rodolfo. (1966). Universidad: Pasado y Presente. Buenos Aires, Editorial Universitaria de Buenos Aires, p. 7.

5 Ver Ongaro Estrada, Andrés A. (2006). La Legislación sobre Educación Superior en el Perú. Lima, Edit. ANR, p.15.

6 Ibid, p.16.
} 
Gobierno Central que debía ser no menos del $20 \%$ del Presupuesto Nacional, y el 6\% de ello correspondería a las universidades.

En cambio, en la Constitución de 1993 ocurre todo lo contrario a la del 79, ya que se limita la gratuidad de la enseñanza y se excluye el $6 \%$ del presupuesto público. Asimismo indica que, con el fin de garantizar la mayor pluralidad de la oferta educativa y a favor de quienes no pueden sufragar su educación, la ley fija el modo de subvencionar la educación privada en cualquiera de sus modalidades, incluyendo la comunal y la cooperativa (artículo 17), artículo que dio sostenibilidad a la proliferación de universidades privadas que sucede hasta el día de hoy. En la actualidad, como bien sabemos, son muchos los problemas que afronta la universidad peruana,y trataremos de analizar los más importantes.

Los orígenes de la universidad peruana se remontan a la fundación de la Universidad Nacional Mayor de San Marcos, el 12 de Mayo de 1551, que fue creada como la asociación de profesores y estudiantes dedicados al estudio, la investigación y la educación, para la difusión de la cultura occidental de ese entonces; en otras palabras, un centro de estudios tradicional, colonial, oligárquico y de honorables, para luego institucionalizarse a inicios de 1919 bajo los intereses de la burguesía agraria-comercial de los terratenientes gamonales. No existía legislación respecto sobre la universidad, porque a inicios del siglo XX solo funcionaban cinco universidades.

Con la Ley $\mathrm{N}^{\circ} 13417$ de 1960 se inicia la denominada "universidad moderna, profesional y reformada", en la que se reconocen las reivindicaciones reformistas como el gobierno universitario, la autonomía, la gratuidad de la enseñanza, etc. En cambio, las leyes universitarias $\mathrm{N}^{\circ} 17437$ y $\mathrm{N}^{\circ} 23733$ llevaron a la frustración a estudiantes y profesores por ser insatisfactoria en los campos científico y técnico; especialmente esta última que se encuentra vigente, en la que se quería adecuar los patrones de dominación de una economía urbana. En la última década, la universidad pierde su fuerza innovadora y movilizadora y hoy nos encontramos con proyectos de la nueva ley universitaria que siguen durmiendo el sueño de los justos ya que, al parecer, no existe voluntad política de promulgar la norma por temor al debate en el sistema universitario y posibles consecuencias que podrían derivar en conflictos internos (universidad) y externos (sociedad).

En su denominación más amplia, la universidad viene a ser una institución de enseñanza superior donde se cursan estudios en las facultades y se confieren los grados correspondientes. Además, la universidad debe ser un centro de tolerancia ante la diversidad de ideas. En ese contexto, se justifica que la generación de investigación y búsqueda de información se convierta en conocimientos y estos, a su vez, sean entregados a los alumnos.

Sus objetivos fundamentales vienen a ser la formación académica, la investigación, la proyección hacia la sociedad, y hoy en día muchos se planteanla nueva educación de la sociedad del saber junto a la tipología de instituciones universitarias en la sociedad del conocimiento (universidades: innovadoras, de docencia diversificada, internacionalizada y virtual) ${ }^{7}$.

Como se mencionó anteriormente, el nombre de universidad deriva de la palabra latina "universitas", que significa el conjunto integral y completo de los seres particulares o elementos constitutivos de una colectividad cualquiera; o sea, la totalidad de una clase o especie de realidades, la que justamente por su carácter universal se distingue de la particularidad de los individuos

Desde otro punto de vista, se define a la universidad como un centro de investigación y docencia al servicio de la sociedad, que aplica sus conocimientos a los problemas reales de la sociedad, y que ayuda a la búsqueda de posibles soluciones desde una perspectiva global, humana e interdisciplinar. Referida a los problemas más urgentes de la región de su influencia, la destacamos en su rol social: "En realidad es un compromiso amplio que se extiende sobre diversos campos vinculados a la reproducción y perfeccionamiento del modelo social: la equidad, la ciencia, la eficiencia profesional, la cultura y la identidad, el pluralismo ideológico, la ética social, la conservación de

7 Rama Vitale, Claudio. (2009). Tendencias de la educación Superior en América Latina y El Caribe en el Siglo XXI. Tomo II Lima, Asamblea nacional de Rectores, pp. 21-42. 
la memoria histórica y de la universalidad del saber, y la creación de masa crítica"8.

Asimismo, en la Conferencia Mundial de la Ciencia para el siglo XXI serecordaba que:"Las universidades deberían velar porque sus programas en todos los campos científicos se centren tanto en la educación como en la investigación y en la sinergia entre ambas. También deberían hacer que la investigación fuese parte integrante de la educación científica y que una parte de la educación de los científicos comprendiese el aprendizaje de las técnicas de relaciones públicas y de las ciencias humanas"9.

1. La situación actual de la universidad podemos sintetizarla en los siguientes

\subsection{Aspectos:}

Estructura universitaria. Antes de 1930 existían nueve universidades públicas y una privada; hoy tenemos en el Perúmás de 129 universidades de diverso tipo; 51 son nacionales (públicas) y 78 son privadas, además de una universidad municipal ${ }^{10}$. Del total de las universidades mencionadas, dos se encuentran sometidas a un proceso de organización. Lima tiene ocho universidades públicas y 32 privadas, es decir 40 universidades y todos los departamentos por lo menos tienen una universidad $^{11}$. De las 51 universidades públicas solo funcionan 35, las demás solo existen en las normas legales. Entre 2006 y 2011, durante el segundo gobierno de Alan García, se crearon 16 universidades públicas de la cuales 12 son simplemente fantasmas y bombas de tiempo; es decir, no existen porque las engendraron mal:“... desafortunadamente se han creado simultáneamente muchas universidades públicas sobre la base de otras filiales, sin presupuesto... "12 y por réditos políticos de algunos congresistas ${ }^{13}$, pero estas doce serán implementadas sobre filiales de universidades o institutos que ya venían funcionando y pronto se convertirán en núcleos de conflicto social. En el año que tiene trabajando el actual gobierno de
Ollanta Humala,ya existen nueve Proyectos de Ley para la creación de universidades esperando ser aprobados; en otras palabras, restarán infraestructura, recursos y profesores a universidades ya existentes. Esta ola incontrolable de universidades es consecuencia del modelo económico de inicios de los años 90 y de la apertura gradual a los mercados internacionales que produjo inversión extranjera directa junto a las nuevas herramientas tecnológicas, generando el crecimiento de la economía peruana y una mayor demanda por parte de las empresas de profesionales con conocimientos cada vez más especializados; es así como surge esta oleada de universidades privadas e institutos superiores tecnológicos.

De otra parte, es de señalar el papel que tiene elConsejo nacional para la Autorización de Funcionamiento de Universidades (CONAFU), que es un organismo autónomo de la Asamblea Nacional de Rectores(ANR) y fue por mucho tiempo el organismo encargado de dar licencias de funcionamiento a las universidades; sin embargo, ante las acusaciones de que no tenía un adecuado control de calidad del servicio educativo, el Tribunal Constitucional lo suspendió de sus funciones e instó al Congreso a crear una Superintendencia que asuma la entrega de autorizaciones a las privadas y supervise el funcionamiento de las públicas(junio del 2010). Luego el Congreso en junio de 2011, ante el vacío administrativo, restituye al CONAFU en sus funciones solo por un año, cumplido el término (junio de 2012)no se sabe quién autorizará el funcionamiento de las universidades que seguiránal borde del abismo.En la actualidad siguen en evaluación del CONAFUvarios proyectos de nuevas universidades, las que no reúnen los requisitos a pesar de que se comenta de una posible moratoria para la creación de más universidades, dizque para homogeneizar la diversidad de normas y de esa manera continúe el desorden y crisis del sistema universitario actual que no sabemos si es coyuntural o estructural; en otras palabras, ante tanta irracionalidad tenemos cantidad y no calidad de

8 Rojas Mix, Miguel.El Compromiso social de las Universidades de América Latina y el Caribe .Declaración del congreso internacional de rectores Latinoamericanos y caribeños Bello Horizonte, Brasil, 2007,Pag 2

9 La Ciencia para el Siglo XXI:Una nueva visión y un marco para la acción. UNESCO, Montevideo, 1998. P. 18.

10 Véase Informe 2011: Dirección de estadística y Planificación universitaria de la Asamblea Nacional de Rectores

11 Según Investigaciones el Ciudadanos al Día (CAD) el mayor crecimiento lo registran las universidades privadas (163\%)sobre la base de información publicada por la ANR en el 2009 existían 98 universidades: 35 públicas y 63 privadas.

12 Ver, Tapia, Marta, Presidenta del Consejo Nacional para la Autorización de Universidades (CONAFU), CONAFU, Enero dl 2012.

13 En los pocos meses del reciente Gobierno, ya seis congresistas han presentado proyectos para crear más universidades, es decir más núcleos de conflictos sociales. En cambio en el anterior gobierno de García Pérez de las 16 universidades creadas durante su gobierno 23 de ellas fueron aprobadas en el último año y medio de gobierno, en otras palabras próximas a las elecciones generales. La forma y fondo como fueron creados se comenta que fueron entre risas y aplausos muchas veces por mayoría simple o unanimidad previa acuerdo, universidades que inclusive ya habían sido descartadas a último momento fueron aprobadas por un rédito político 
universidades en comparación con otras latitudes, que son inexistentes en los ranking mundiales y continentales. Tenemos más que suficientes instituciones ${ }^{14}$ para cubrir la demandada de estudiantes, el problema es la falta de calidad y ello no se soluciona creando más universidades ${ }^{15}$.

Población. En 1960 se matricularon 30,000 alumnos aproximadamente, y en la actualidad, según la ANR, contamos con más de 700,000 estudiantes y cada año ingresan alsistema universitario un promedio de 90,000 estudiantes. Según el último censo universitario, el número de estudiantes de pregrado de las 35 universidades públicas se aproxima a los $8^{\prime} 000,000$,distribuidos en más de 200 carreras profesionales, y en el post grado, son alrededor de 25,000 estudiantes. Entre las universidades que ofrecen mayor número de Maestrías estarían la Universidad Nacional Mayor de San Marcos (87); Universidad Nacional de San Agustín (76); Universidad Nacional de Trujillo (71). En las universidades privadas tenemos ala Pontificia Universidad Católica del Perú (52); Universidad de San Martín de Porres (45); Universidad Inca Garcilaso de la Vega, con 44 maestrías. Es de mencionar que estos datos son relativos, por lo mismo que los post grados son autofinanciados, y se aperturany cierran menciones de acuerdo a las necesidades del mercado.

El cuanto al número de postulantes, las universidades públicas recibieron casi el doble que las universidades privadas. Las universidades privadas tienen mayor número de graduados y titulados, yde los más de 300,000 alumnos matriculados en universidades públicas en 2012 , el $58 \%$ eran hombre y el $42 \%$, mujeres.

Presupuesto. Cómo vamos a enfrentar el déficit presupuestal de las universidades para que cumplan los objetivos para los que fueron creadas es uno de los problemas principales, ya que el gobierno brinda escaso apoyo para incrementar la inversión en educación, y la mayoría de las universidades subvencionan los estudios ya que los pagos por derecho de matrícula son casi simbólicos. En términos generales del presupuesto asignado a las universidades, del total, el $80 \%$ de estos presupuestos están destinados a pagar remuneraciones, siendo la partida más descuidada la de bienes y servicios que siguen deteriorándose desde hace tres décadas. En mayo del 2004 se realizó el denominado Pacto Social de Compromisos Recíprocos por la Educación 2004-2006 (gobierno, partidos políticos, gremios empresariales y laborales, sociedad civil, etc., reunidos en el Foro del Acuerdo Nacional). El Pacto ratifica el compromiso de incrementar anualmente el $0.25 \%$ del PBI hasta que la educación alcance el $6 \%$ ya que ahora apenas se aproxima al 3\% del PBI. Sin embargo, el presupuesto de Educación del 2012 no incorpora dicho incremento; asimismo según fuentes del Ministerio de Educación, no progresa la inversión en educación desde el año 2000, a pesar del crecimiento de la economía y del crecimiento de la presión tributaria en su proyección de alcanzar el 16\% en el 2012, sin contar el gravamen minero ni regalías debido a que tales conceptos no son considerados propiamente impuestos. Las universidades públicas que recibieron mayor dinerosegún el presupuesto aprobado para el año 2012 son la Universidad Nacional Mayor de San Marcos (S/. 360,598,804), la Universidad Nacional de San Antonio Abad del Cusco $(S / .159,550,602)$ y la Universidad Nacional de Ingeniería (S/.219,532,038). En el otro extremo del presupuesto se encuentran las Universidad Nacional de la Frontera, la Autónoma de Chotay la Intercultural de la Selva Central Juan Santos Atahualpa, las que reciben $\mathrm{S} / .3,500,000$, que es el mismo monto que recibieron en el presupuesto de 2011.En cuanto a las fuentes de financiamiento de las universidades públicas, más o menos el $58 \%$ de su presupuesto asignado proviene de la recaudación tributaria del estado. El 30\% corresponde a recursos directamente recaudados por las mismas universidades, es decir tasas, ventas de bienes, prestaciones de servicios, entre otros;

14 Véase al respecto Delgado de la Flor Badaraco, Francisco y Alcázar C., Marcia. (2004). Informe sobre Educación Superior Universitaria. Lima, Asamblea Nacional de Rectores. Ver, Aguirre Yato, Víctor y otros. (2008). Estrategias para el desarrollo de las Universidades del Perú. Lima, Asamblea Nacional de Rectores.

15 Curiosamente es de mencionar que mientras en el Perú se crean universidades sin ningún criterio académico, en los países vecinos como Ecuador donde cierran 14 universidades donde estudiaban 38,000 alumnos, por falta de calidad académica, previa evaluación durante 18 meses por el Consejo de Evaluación de la Educación Superior. Siete de las universidades son de la capital y el resto de diversas provincias. Las universidadesclausuradas son lassiguientes: Universidad Autónoma de Quito, Cristiana Latinoamericana Intercontinental, Alfredo Pérez Guerrero, Panamericana de Cuenca, Interamericana del Ecuador, OgMandino, Técnica San Antonio de Machala, TecnológicaAmérica, UniversitasEquatorialis, Técnica Particular de CienciasAmbientales José Peralta, la Escuela Superior PolitécnicaEcológicaProfesorServioTulio Montero Ludeña, la Escuela Superior PolitécnicaEcológicaAmazónica y la EscuelaPolitécnicaJavierana. 
9\% responde a donaciones y transferencias, y $3 \%$ a canon ${ }^{16}$ y sobrecanon, regalías, rentas de aduanas y participaciones ${ }^{17}$. Es de remarcar que no todas las universidades reciben canon minero, solo aquellas ubicadas en los departamentos donde existe actividad minera.En términos comparativos, somos uno de los países en Latinoamérica que menos gasta en educación por habitante. Según fuentes de la Comisión Económica para América Latina y el Caribe (Cepal), el Perú invirtió US\$ 58 al año por habitante; Bolivia, US\$ 66; Colombia, US\$ 97; Brasil, US\$ 185, y Chile, US\$ 238. Y, en el caso de las 30 universidades públicas, estas reciben en conjunto la mitad de los que gasta una universidad pública en los Estados Unidos. En resumen, no tenemos autonomía económica y seguimos atados a la burocracia estatal en el manejo del dinero.

Democracia. La actual democracia elitista y excluyente no es representativa ni es suficiente. Las elecciones de las autoridades, como el rector, vicerectores, decanos de facultades y de escuela de post grado, así como el propio comité electoral, deberían ser por voto universal, aunque ello no resuelva el problema de la universidad. El sistema de elección universal y secreta en las universidades impediría la instalación de cúpulas acuarteladas en el rectorado y decanatos, y garantizaría la práctica de la democracia, ya que la universidad es una tribuna libre de ideas que ayuda a transformar la sociedad. Practicar la democracia no es politizar o generar el desorden en la universidad es, más bien, todo lo contrario. Asimismo con esta ley las escuelas de postgrado no se sienten representadas ni tienen participación y decisión en el gobierno de la universidad; es decir, no participan en los consejos de Facultad, Asamblea Universitaria, Comisiones de Gestión, tan solo tienen representación mediante el Director de la Escuela de Postgrado con un voto solitario ante su problemática académica y administrativa, y más parecieran inquilinos precarios pese a que, con el tiempo, se han convertido en la caja chica de los gobiernos universitarios de turno.

Calidad. Al hablar de calidad nos referimos a la condición que tiene todo servicio público o privado de la educación y, en particular, a la manera como se brinda ese servicio, en este caso la docencia, investigación y la proyección social. En el sistema universitario no existe un organismo especializado de acreditación para las universidades, con capacidad de decisión y seguimiento del conjunto de normas y procedimientos estructurados que puedan definir y establecer los criterios, estándares y procesos de evaluación, acreditación y certificación, con la finalidad de asegurar los niveles básicos de calidad que debe brindar una institución universitaria. En otro ámbito tenemos al amparo de la Ley de Educación,el Sistema Nacional de Evaluación y Certificación de la Calidad Educativa (SINEACE) $)^{18}$. Un estudio realizado ${ }^{19}$ por SINEACE indica que la educación superior es muy heterogénea, con un límite inferior bajo,lo que se debe -principalmente- a que las instituciones de educación superior no tienen incentivos ni recursos suficientes para invertir en calidad académica-administrativa; en este caso las universidades y sus escuelas de postgradosolo lograrán su calidad previa aplicación de un modelo de autoevaluación sostenida como estrategia de mantenimiento en camino a su acreditación nacional e internacional. Hasta la fecha, las cifras son desalentadoras: solamente 31 carreras profesionales son las que llegaron a acreditarse; por lo tanto, la autoevaluación y acreditación, tal como están planteadas, son un fracaso. Es de remarcar que todo esto se sigue arrastrando porque existe una fisura dolorosa entre la educación secundaria y la universidad, por el desfase y los objetivos que se buscan. Mientras que en la educación secundaria $^{20}$ la formación principalmente es metodológica (hábitos de estudio, saber estudiar, entre otros)

16 La ONG Ciudadanos al Día (CAD) informa que al presupuesto aprobado para el período 2012 se añadirán US\$ 336 millones por concepto ce canon, tributo correspondiente al $20 \%$ del total de ingresos y rentas obtenidos por el Estado por la explotación de yacimientos mineros, petroleros y gasíferos. Estos fondos van actividades de investigación y desarrollo de ciencia y tecnología en las universidades públicas de las zonas de influencia de dichos recursos naturales. Ver, Informe. CAD, enero del 2012.

17 Según Ciudadanos al Día (CAD), en la Educación los gobiernos regionales tienen que asumir más responsabilidades como incentivar los servicios educativos; promover la educación intercultural y el uso de lenguas de la región; formular políticas de educación, ciencia, tecnología, deporte y cultura; promover la declaración de bienes culturales no reconocidos de la región; así como diseñar y evaluar el proyecto educativo de su jurisdicción. Deben también implementar políticas de infraestructura y equipamiento; proteger y conservar el patrimonio cultural nacional; gestionar los servicios básicos de educación básica y superior no universitaria, así como los servicios educativos.

18 Sistema Nacional de Evaluación, Acreditación y Certificación de la Calidad Educativa. Se refiere a la Ley de General de Educación N²8044 quebuscapromover el desarrollocualitativo de la calidad de la educación.

19 Estudio realizado por el SINEACE sobre la Educación Superior en el Perú: Retos para el aseguramiento. 2010.

20 Ver Carvallo, Constantino. (2009). Diario Educar. Lima, Editorial Aguilar; Cisneros, Jaime Luis. (2009). Aula Abierta. Lima, Editorial Norma; Penac, Daniel. (2008). Mal de escuela. Ciudad, EditorialMondadori; Mc Court, Frank. (2007). El profesor. Ciudad, Editorial Norma. Estos autores indistintamente expresan el sistema educativo desde diferentes puntos de vista. 
junto al aprendizaje del saber; en otras palabras, ayuda a no pecar de ignorancia en un mundo moderno, competitivo y global. En cambio, en la universidad se persiguen otros objetivos como la investigación, la formación profesional y su proyección social hacia la sociedad. Es importante destacar que, hasta la fecha, ninguno de los dos niveles de educación mencionados cumple los objetivos para los que han sido creados.

Investigación. La inversión en investigación no solo es insuficiente sino casi nula. El Perú invierte el $0.1 \%$ de nuestro PBI en este rubro, mientras que Brasil invierte 1.1\%, Estados Unidos,el 2.7\%, y Finlandia, el 3.5\%. El debate está, de un lado, en que el Estado con los recursos de todos los peruanos se asigne la potestad -que no le corresponde- de elegir qué proyectos de investigación financiará directamente, pese a que la investigación cada vez necesita más ala empresa privada para investigar y producir mejor por la interacción del mercado que requiere de innovaciones tecnológicas de acuerdo a las exigencias del país y del consumidor (solo un 2\% de las empresas peruanas invierte en investigación hoy en día). El Estado podría generar incentivos(por ejemplo tributarios) para que los empresarios inviertan más de sus propios recursos en investigación y desarrollo; de otro lado, el debate continúa sobre la propuesta de crear un Ministerio de Ciencia, Tecnología e Innovación o reforzar el Consejo Nacional de Ciencia, Tecnología e Innovación Tecnológica (CONCYTEC) ${ }^{21}$.Otro de los problemas no resueltos es la insuficiencia de investigación y recursos humanos (investigadores) a dedicación exclusiva con categoría de profesor ordinario investigador ${ }^{22}$. El país necesita producción diversificada y de alto valor agregado, lo que se lograría con la educación e inversión en ciencia y tecnología; para esto tenemos la articulación del trio: Universidad-Estado-Empresa,que es una prioridad aunque es poca o casi nula la vinculación con la empresa privada en relación a la investigación, desarrollo e innovación científica y tecnológica.En este contexto, el mejoramiento de las condiciones generales no ha sido suficiente para superar su situación. Muestra de ello son los bajos niveles de relación entre nuestras organizaciones, el escaso intercambio de experiencias que tenemos, la prácticamente inexistente movilidad docente y estudiantil, y sobre todo, la casi total ausencia de investigaciones y tecnologías necesarias para cumplir con nuestra misión: generar mayores oportunidades tanto en beneficio de las personas que formamos, como para contribuir al desarrollo social y al aumento del bienestar de nuestra población.

Normatividad jurídica. En términos generales, la estructura y funcionamiento de la educación superior se basa en la Ley General de Educación, Ley $\mathrm{N}^{\circ} 23384$, que incorpora a la educación inicial. primaria, secundaria y superior, comprendiendo está ultima los Institutos Superiores Tecnológicos y Pedagógicos. En cambio las universidades están reguladas por la Constitución Política de 1993 (Capítulo II, artículo 18) y la Ley Universitaria, Ley $\mathrm{N}^{\circ} 27733$ ), que define que son autónomas en su régimen académico, administrativo y económico, su sistema de admisión, vacantes, carreras profesionales que ofrecen, entre otras. Algunas universidades privadas se rigen por la Ley de Promoción de la Inversión en Educación, Decreto Legislativo $\mathrm{N}^{\circ} 882$ que busca incentivar la participación privada en el sector educación ante la demanda que existe en la sociedad. Antes de la promulgación de esta norma, las universidades tenían los mismos fines y estructura académica y administrativa equivalente y, en el ámbito del financiamiento, las universidades públicas eran financiadas por el Estado y las privadas por particulares. Hoy, después de este Decreto Legislativo cualquier persona natural o jurídica, realizando los trámites pertinentes y sin tener ninguna formación académica, puede fundar una universidad con fines de lucro, mercantilizando y proliferando desmesuradamente la creación de universidades sin calidad educativa.

21 El CONCYTEC viene a ser la instituciónrectora del SistemaNacional de Ciencia,Tecnología e InnovaciónTecnológica (SINACYT), integradopor la Academia, los Institutos de investigación del Estado, lasorganizacionesempresariales, lascomunidades y la sociedad civil. Estáregidopor la Ley Marco de Ciencia y Tecnología, Ley N²8303, que lo estableció en el DecretoSupremo $\mathrm{N}^{\circ} 082-2005$-PCM, referente a suadscripción al Ministerio de Educación y la Ley del CONCYTEC. Ver Portal del CONCYTEC, www.concytec.gob.pe.

22 Existe un proyecto de creación del Ministerio de Ciencia y Tecnología para un mejor trabajo de investigación científica.

23 Wong Torres, Zelma. (2004). "El Idioma Chino, lengua del futuro". En Revista Quipukamayoc, Facultad de Ciencias Contable de la Universidad Nacional Mayor de San Marcos. Lima, Editorial UNMSM, pp. 35-38.

24 Al respecto de las variables economía de mercado y universidad, ver Claudio Rama, Director del Instituto Internacional de la UNESCO para la Educación Superior en América Latina y El Caribe (IESALC). Ver "Ética y educación superior en el contexto de la mercantilización", en línea: www.iesalc.unesco.org.ve/.../Art \%EDculo\%20-\%20Etica\%20y\%20educaci\%F3n\%20superior.pdf 
Mercado laboral. La universidad, en general, no responde a las exigencias y necesidades de las empresas públicas y privadas en el país. No se prioriza que áreas del conocimiento y el desarrollo se requiere priorizar y en qué áreas se necesitan o se requieren profesionales de acuerdo a las exigencias del mercado y el desarrollo nacional. Ahora bien, es de mencionar que está bien que la juventud estudie y se capacite pero cuidando la calidad en su formación y enseñanza para que, al término de sus estudios,el alumno pueda insertarse laboralmente en este mercado global emergente y competitivo, y no emigrar como viene sucediendo ya que un tercio de todos los científicos formados en el tercer mundo no trabajan en sus países y el 50\% de los doctorados que viajan a Estados Unidos y Europa no regresan a sus países de origen. La emigración de científicos, intelectuales y profesionales de nuestro país se debió al escenario de violencia política que vivimos años atrás. Todo ello no solo resta calidad a la formación, sino que debilita su papel como institución generadora de conocimientos y vinculada al desarrollo económico político y social de su entorno inmediato, y del país en general.

Número de egresados. Los egresados no corresponden al número de graduados con tesis en pre grado y post grado. Ante los bajos índices de investigación, se propone que el estudiante ya no opte por el examen de grado sino por una tesis productiva, aunque el artículo 23 de la actual Ley Universitaria da la posibilidad de obtener el título de licenciado o sus equivalentes mediante la presentación de una tesis o un examen profesional. Por citar un ejemplo, el 80\% de ingresantes a una Maestría o Doctorado no llegan a graduarse por diferentes aspectos, entre ellos, fundamentalmente, por la falta de certificación del idioma y la falta de metodología de investigación, en otras palabras superemos el síndrome del TMT (todo menos tesis).

Manejo de Idiomas. La exigencia científica no solo demanda el manejo de idiomas sino también la demanda laboral como parte de la globalización de la ciencia y las universidades, así como en el ejercicio profesional, cultural y hasta deportivo; por lo tanto, el idioma no dejade ser una referencia en la inserción en el mercado laboral nacional e internacional que cada día es más exigente. Ya no es sostenible tener una carrera con el manejo intermedio de un idioma; se prevé que en el mundo, aunque es imposible dar una cifra exacta de cuántos idiomas se hablan, se calcula que en la actualidad existen más de 4,000 lenguas y el idioma que dominaría el mundo-aunque por ahora es imposible saberlo si reemplazará totalmente al Inglés- sería el Chino Mandarín ${ }^{25}$, porque ya es utilizado por más de 900 millones de personas(casi dos veces más que el inglés) y por la importancia comercial y económica que tienen en la escena internacional. Quien domina idiomas tiene una ventaja sumamente valiosa frente a otros profesionales con formación similar.

El rol de los intelectuales y la universidad. En su formación académica la universidad debe brindar instrumentos de conceptualización y teorización que estén al alcance de los participantes, que les permita pensar, reflexionar y analizar objetivamente y con libertad.Una universidad no cumpliría su rol si descuidara la búsqueda libre del conocimiento y su aplicación a la realidad social de su país, región o localidad. El intelectual o creador, con sus opiniones, genera corrientes de opinión, por eso decimos que en un país no todo es economía de mercado, sino que tiene que existir la otra parte que es la ética y reflexión que es el rol que tiene la universidad y los intelectuales como conciencia crítica de una sociedad, asunto que hoy las economías más inteligentes entienden ya que el conocimiento se tiene que convertir en Producto Bruto Interno (PBI).

Credibilidad. Si tenemos que recuperar la credibilidad de las universidades, es necesario que triunfe la meritocraciay la idoneidad; para esto sería importante empezar con transparencia ${ }^{26} \mathrm{el}$ ingreso y nombramiento, ascensos y ratificaciones decatedráticos. Las facultades en las universidades deben entrar en un proceso de reestructuración y reforma integral con una reingeniería en la selección de profesores, invitando a los mejores catedráticos; de esta manera, el proceso deenseñanzaaprendizaje corresponderá con los objetivos de las facultades, talleres y tesis de investigación, y la malla curricular responderáal perfil y a las nuevas exigencias profesionales. Asimismo, si seguimos hablando de transparencia ${ }^{27}$, por citar un ejemplo,

26 Ver la Ley N²7806, Ley de Transparencia y Acceso a la Información. Esta ley viene a ser un documento que regula las instituciones públicas sobre la información pertinente y de carácter público de las actividades y servicios que brinda.

27 Al respecto de las variables economía de mercado y universidad, ver Claudio Rama, Director del Instituto Internacional de la UNESCO para la Educación Superior en América Latina y El Caribe (IESALC). Ver "Ética y educación superior en el contexto de la mercantilización”, en línea: www.iesalc.unesco.org.ve/.../Art \%EDculo\%20-\%20Etica\%20y\%20educaci\%F3n\%20superior.pdf 
sería importante empezar con una auditoría académica como muchos académicos lo plantearon, donde se pueda revisar los catálogos de los títulos y grados universitarios y, de esta manera, se pueda revisar la transparencia de los procedimientos de validación de las fuentes de información de las tesis; así podremos ver si cumplen con estándares determinados por las normas Vancouver ${ }^{28}$ y se podrá contrastar la originalidad de los trabajos de investigación.

Por último, y lo que más nos preocupa son tres aspectos de mucha trascendencia en la universidad, nos estamos refiriendo a la gestión, liderazgo y cultura organizacional.

En relación a la gestión ${ }^{29}$, es el talón de Aquiles de las universidades por la falta de capacidad de gestión de los rectores, vicerrectores, decanos y personal directivo en sus diferentes instancias, sumándose a esto la falta de seguimiento de los objetivos, estrategias, proyectos y la falta de información para la toma de decisiones de los responsables o regentes.En términos generales, las universidades no gestionan su conocimiento knowhow y no es transferida la información a los responsables o regentes de la universidad. En el ámbito económico se deben incrementar los mecanismos y medidas de control para la transparencia y rendición de cuentas en todos los niveles, que es una de la formas de impedir la corrupción en la administración de los recursos y en camino hacia una mejora de la gestión universitaria.

Sobre el liderazgo, la mayoría de los responsables tienen un liderazgo de interés de grupo con un estilo marcadamente de caudillismo y lo que necesita la universidad es un liderazgo con capacidad de influenciar y guiar a individuos o grupos con valores, acciones, resultados, conocimientos y con una visión común del rol de la universidad a nivel local, regional y global.

La cultura organizacional ${ }^{30}$ obedece a motivaciones interesadas o controlistas por parte de algunos, lo que enrarece el clima democrático y dificulta el grado de conocimiento y valoración de los elementos de la cultura organizacional de sus respectivas universidades, influyendo esto de forma negativa en el clima laboral."Las universidades del país no tienen claro o no realizan una gestión de cultura organizacional. La cultura que poseen es producto de la intervención del azar en la actividades de la universidad, de los hábitos y costumbres de sus miembros, y la influencia de una personalidad importante". Muchas veces la universidad crececuantitativamente (aulas, alumnos) y cualitativamente va disminuyendo su capacidad para optimizar su cultura organizacional como por ejemplo, los enfoques de calidad ${ }^{31}$.

3. Perspectivas de la universidad y las escuelas de post grado.

En primer lugar, antes las universidadespodían evaluar y acreditar su calidad mediante diferentes criterios y sistemas de acreditación, como el norteamericano y el de la Asamblea Nacional de Rectores $^{32}$, a través del Instituto de Investigación y Fomento de la Modernización y Acreditación Universitaria (ANR-INFOMACU, 2001). Hoy la acreditación de la calidad educativa se basa en la Ley $\mathrm{N}^{\circ} 28740$, Ley del Sistema de Acreditación y Certificación de CalidadEducativa (SINEACE) y

28 El origen de las normas de Vacouver: el Comité Internacional de Directores de Revistas Médicas se reunió informalmente en Vancouver, Columbia británica, en 1978, para establecer las directrices que en cuanto a formato debían contemplar los manuscritos enviados a sus revistas. El grupo llegó a ser conocido como Grupo Vancouver. Sus requisitos para manuscritos, que incluían formatos para las referencias bibliográficas desarrollados por la National Library of Medicine (NLM) de EstadosUnidos, se publicaron por vez primera en 1979. El Grupo Vancouver creció y se convirtió en el Comité Internacional de Directores de Revistas Médicas (CIDRM), que en la actualidad se reúne anualmente.

29 Al respecto, SusanChen (2004) define como gestión del conocimiento “... al proceso de gestión organizacional cuyo objetivo es identificar el conocimiento que producen los empleados de alto desempeño de una empresa para convertirlo en información que pueda ser re-utilizada por el resto de los empleados de esa empresa. El objetivo fundamental de este proceso consiste en identificar el modo en que las personas utilizan la información para generar nuevos conocimientos, las competencias que ponen en juego para ello, los procesos de comunicación informal, la capacidad de trabajo en equipo, y la motivación que las personas pueden tener o no para compartir sus conocimientos". Ver en Revista de las Sedes Regionales de Costa Rica. Intercedes, Costa Rica Vol V. N7, 2004. En http://www.intersedes.ucr.ac.cr/07-autores. html\#autor06. Consulta hecha el 21 de noviembre del 2007.

30 Según Robbins, la cultura desempeña numerosas funciones dentro de la organización, destacando las siguientes: crea distincionesentre una organización y las demás; transmite un sentido de identidad a los miembros de la organización, facilita la generación de un compromiso con algo más grande que el interés personal del individuo, incrementa la estabilidad del sistema social, proporciona los estándares sobre los que deben decir y hacer los empleados y sirve como mecanismo de control y sensatez que guía y moldea las actitudes y el comportamiento de los empleados. Ver, Robbins, Stephen, P. (1996). "Cultura organizacional”. En: Comportamiento Organizacional. México, Prentice Hall Hispanoamericana, pp. 437-453.

32 Véase Aguirre Yato, Víctor y otros. (2007). Estrategias para el desarrollo de las Universidades del Perú. Lima, Asamblea Nacional de Rectores.Comisión Especial, designada por Resolución N³68-2007-ANR, p. 21 
su respectivo reglamento ${ }^{33}$. Existen otros tipos de acreditación en el ámbito académico, como:

Acreditación especializada por programa. Mediante este mecanismo se acreditan generalmente carreras que tengan relación e impacto con la salud, la seguridad y el bienestar social.

Acreditación institucional. Por el que se acreditan las características. En general, se evalúan los recursos, servicios, el proyecto educativo, la capacidad de gestión y la situación financiera, entre otros. Se dice que mayormente es complementaria y posterior a la acreditación por programas.

Acreditación de profesionales. Permite autenticar la competencia técnica de los titulados de un programa para el ejercicio profesional, responsabilidad casi siempre asumida por las asociaciones profesionales quienes establecen los mecanismos para evaluar.

Bien, entonces antes de definir la autoevaluación, podemos decir que la acreditación se consigue como resultado del desarrollo de tres momentos básicos:

- La autoevaluación.

- La evaluación por pares externos.

- La acreditación.

La Universidad Nacional Mayor de San Marcos define la autoevaluación como una etapa del proceso de acreditación en la que la propia institución se evalúa internamente, según normas o estándares de calidad que tienden a ser de alcance internacional, con el objeto de determinar su cumplimiento o incumplimiento, especificando las áreas en las que se requiere mejorar. Esta actividad la desarrollan comités nombrados por la institución o unidad académica, los cuales realizan su tarea mediante guías previamente definidas, coordinando la recopilación de la información, su análisis y la elaboración del informe de autoevaluación, todo esto junto a la motivación interna, el respaldo institucional y el liderazgo y participación plena de todos los miembros de la Universidad ${ }^{34}$, valores sin los cuales no sería exitoso el proceso de autoevaluación.

Esto es, este proceso se convierte en un componente fundamental para poder describir, emitir o validar juicios de valor sobre la institución, o nos ayuda a examinar planes, acciones y logros, comprobar e interpretar los logros de un programa y/o mejorar un programa o curso de acción para que responda a las necesidades de un mercado laboral, en la búsqueda del desarrollo económico y social del país.

En términos generales, todo proceso de evaluación de un programa de acreditación ${ }^{35}$ presupone, entre otros, definir los propósitos de la evaluación; delimitar sus variables, elementos, indicadores y criterios, para establecer el nivel y calidad del programa de funcionamiento, así como recolectar, analizar e interpretar información pertinente; valorar la calidad del programa en su totalidad o en algunas de sus variables y elementos; analizar el logro del programa evaluado; identificar e incorporar los ajustes y cambios pertinentes; y establecer los procesos permanentes de seguimiento y control.

En el caso de las escuelas de postgrado, según opiniones de instituciones y otros expertos, se pueden medir por diferentes modalidades como la autoevaluación interna y externa. En el primer caso, la organización, ejecución y supervisión del programa de autoevaluación tiene que estar bajo responsabilidad de personas que tengan el interés y compromiso con el programa. En el segundo, participarán especialistas ajenos al programa, identificando los problemas, las dificultades y sugiriendo más no haciendo correcciones al programa ${ }^{36}$. Si como proceso global la autoevaluación sirve para medir las fortalezas y debilidades de una institución entonces, para llegar a ese objetivo, tenemos que apoyarnos en un conjunto de factores propuestos en un modelo de matriz de autoevaluación, que luego pueden ser agrupados en criterios e indicadores de gestión de calidad,

33 Véase, Asamblea Nacional e Rectores. Comisión Nacional de rectores para la acreditación, Dirección general de Investigaciones y acreditación Universitaria. Dcto. De trabajo. Estándares Mínimos para la Autoevaluación de las carreras profesionales; Edit.ANR, Lima, Perú, 204

34 Ley 28740, promulgada el 19 de Mayo de 2006 y publicado su Reglamento el 10 de Julio de 2007.

35 Ver Universidad Nacional Mayor de San Marcos. (2006). Lineamientos para una Política de Calidad, Autoevaluación y Acreditación en la UNMSM. Documento de Trabajo,p.73.Véase, GutiérrezHuby, Ana María. (2006). "Formulación y desarrollo de un modelo de autoevaluaciónpara la acreditación del Programa de Contabilidad". Tesisparaoptar el Grado Académico de Magíster en Administración, mención Gestión Empresarial. Lima, UNMSM, p.32.

36 Al respecto véasetambién, Cabrejos Polo, Jorge; Gutiérrez Huby, Ana maría y LópezMás, Julio. (2006). Guía de Autoevaluaciónpara la acreditación en el area economic-empresarial. Lima, Editorial UNMSM. Ver, Oficina Central de CalidadAcadémica y Acreditación de la UNMSM. (2005). Hacia la Autoevaluación en San Marcos. Lima, Editorial UNMSM. 
entonces surge la interrogante:¿qué son y para qué sirve los indicadores en la autoevaluación?

Podemos decir que un indicador viene a ser un elemento de medición que permite aclarar y medir en el tiempo un concepto; laexpresión indicador también se refiere a los datos cuantitativos y cualitativos (números, medidas, opiniones, hechos, percepciones) que nos ayudarán a tener en cuenta lo que pueda estar sucediendo en el ámbito o sector que nos interesa conocer. Asimismo, decimos que sirve porque nos permitirá medir los cambios o situaciones a través del tiempo. Estos instrumentos son importantes también porque, a través de ellos, podemos evaluar y dar inicio a los procesos de desarrollo para alcanzar y orientar mejores resultados en la autoevaluación.

En cambio, la acreditación es el reconocimiento formal y público, por parte de un organismo oficial autorizado, de la competencia técnica de una entidad para la realización de una actividad perfectamente definida. Se trata, pues, de un proceso a través del cual se busca comprobar que la institución por acreditar desarrolla sus actividades en función de su plan estratégico, además de certificar periódicamente el cumplimiento de un conjunto de estándares mínimos de calidad previamente establecidos. Consecuentemente, la acreditación propicia el mejoramiento de la calidad, idoneidad, pertinencia y solidez de la institución, e incentiva en sus integrantes una cultura de evaluación permanente ${ }^{38}$.

Entre los objetivos que busca la acreditación, principalmente, está el ser un mecanismo para que las instituciones de educación superior rindan cuentas a la sociedad y el Estado, dar fe pública de la calidad de las instituciones y de los programas de educación superior; asimismo, sobre el servicio educativo que prestan, brindar información confiable a los usuarios y ser un instrumento para que las instituciones verifiquen el cumplimiento de su misión, sus propósitos, objetivos, en el marco de la Constitución y la ley, y de acuerdo a sus estatutos.
En ese sentido implica certificar públicamente los requerimientos mínimos de calidad que reúne un programa o una institución académica sin la cual no podría mejorar el sistema universitario del país; en otras palabras, la acreditación tiene como propósito garantizar la calidad de las instituciones o programas de la universidad y ayudarlos a superar sus niveles de calidad con el mejoramiento continuo, entendiendo el mejoramiento como una filosofía que ha rebasado al ámbito empresarial y que se aplica también en el sector educativo, por lo mismo que todos pretendemos progresar hacia el autoexamen mediante mejoras graduales y constantes que brotan como iniciativas de las actividades diarias y que son parte de las acciones frecuentes en el ámbito laboral. Al margen de las definiciones, lo que nos interesa es que se desarrolle una cultura de mejoramiento continuo(elemento indispensable para el mejoramiento) en todos los estamentos (profesores, estudiantes y administrativos)de la universidad, desterrando la soberbia organizacional, el miedo al cambio, la falta de tolerancia y la poca participación del personal ${ }^{39}$.

En esa orientación se pueden medir indicadores y criterios para establecer el nivel de calidad del programa en vigencia; la gestión académica y administrativa de la universidad y sus postgrados; grados académicos de sus profesores; selección y admisión de alumnos; las distintas responsabilidades que asumen los graduados en las instituciones públicas y privadas de la sociedad; el currículo; el impacto social; la investigación; número de publicaciones especializadas; horas de atención a los alumnos; infraestructura de sus aulas, bibliotecas, laboratorios, cafeterías, instalaciones deportivas; sistema de apoyo para la búsqueda de apoyo, entre otros. En resumen, se busca medir la eficiencia en la organización académica y de gestión dela universidad y el postgrado.

Ahora bien, para poder acreditar la calidad educativa $^{40}$, se tienen que plantear objetivos dentro de un plan estratégico como formar profesionales

37 Véase Consorcio de Universidades. (1999). Autoevaluación institucional: Manual para Instituciones de educación superior. Lima, Editorial Fondo Editorial Universidad del Pacífico, pp. 58 y ss.

38 Ver Universidad Nacional Mayor de San Marcos. (2006). Lineamientos para una Política de Calidad, Autoevaluación y Acreditación en la UNMSM. Documento de trabajo, p.76.

39 Sobreacreditacióntambién se puedever: De Paz Toledo, Zenón y Cuba Sancho, Juana. (2003). Lineamientosparaunapolítica de calidad, autoevaluación y acreditación. Lima, Editorial UNMSM. Véase, Consorcio de Universidades. (1999). Autoevaluación institucional: Manual para Instituciones de educación superior. Lima, Editorial Fondo Editorial Universidad del Pacífico.

40 Yarzábal, Luis. (2002). "La Educación Superior en la sociedad contemporánea”. Conferencia dictada en el Primer Foro Nacional de la Educación Superior. Managua, mayo del 2002, En: Universidad y Sociedad, revista del Consejo Nacional de Universidades, $N^{\circ} 10$, septiembre del 2002. Managua, p. 8. 
de altísima calidad; la internacionalización con soportes de alianzas estratégicas con otras universidades; mejorar el posicionamiento en cuanto a los competidores; desarrollar programas de investigación con participación de docentes y estudiantes y/o en convenios de cooperación nacional e internacional; modernizar las currículas de las facultades y los syllabus de los cursos con un adecuado apoyo; modernizar la gestión con diferentes acciones a tomar en función de las políticas sobre administración, eficiencia y productividad; ética profesional, elemento fundamental para generar conciencia de sus responsabilidades en su desarrollo social, la producción y el fortalecimiento de una cultura organizacional; formar líderes con una visión compartida y una cultura institucional de acuerdo a la exigencia de los cambios en la escena de investigación, la rigurosidad, la tecnología y el desarrollo de competencias; incorporar en su gestión estratégica universitaria la necesidad de vincular a la universidad con la sociedad, no solo a nivel de sus representantes, sino de toda la comunidad universitaria, generando acciones institucionales que respondan a las necesidades de toda la sociedad.

Podemos decir, entonces, que las universidades y sus escuelas de post grado deben ser dinámicas, creativas e innovadoras, para que junto a la tecnología de las comunicaciones permitan acercar la calidad de investigación, de enseñanza y de gestión. En esa orientación diríamos que no metamos cerrojo a las puertas del cambio porque estaríamos negando toda posibilidad de progreso.

Antes de concluir,quiero señalar que las lecciones aprendidas del pasado son importantes, como dice el refrán: "uno aprende y después desaprende para aprender solo"; en ese sentido,les invitamos a tener una visión compartida hacia un proyecto nacional de universidad y de escuelas de postgrado; a actuar y comprometer nuestras voluntades para superar la situación actual de la universidad y para afrontar la competitividad, así como el fomento y exigencia de la modernización a través de la autoevaluación y acreditación con el propósito de garantizar la calidad de las universidades y programas del postgrado en el Perú.

Sirvan pues, estas reflexiones para reiniciar el debatesobre las universidades y escuelas de post grado, ya que desde la recuperación de la democracia, se ha esperado que algunas universidades se fortalezcan y otras reconstruyan su potencial en términos de docencia, investigación, publica- ciones, y la vinculación con la sociedad a través de actividades de extensión y transferencia de tecnología, aunque los problemas siguen siendo económicos y de rentas, entre otros.

Para finalizar, podemos afirmar una vez más, que launiversidad cumple un rol fundamental en la sociedad del conocimiento y no podemos estar de espaldas a la realidad;todavía quedan muchas interrogantes en el tintero como por ejemplo: ¿cuál es el compromiso social de la universidad frente a los nuevos enfoques de desarrollo científico, tecnológico y de gestión del conocimiento, en un contexto de globalización de las economías de mercado?, ¿seguiremos pensando sin dar nuevos lineamientos sobre el nuevo contexto social, económico y político en el que se encuentra la universidad peruana?,¿a qué distancia se encuentra la universidad latinoamericana frente a los avances en ciencia, tecnología y gestión de conocimientos en los países desarrollados y emergentes?, ¿cuáles son las estrategias impulsoras del cambio de paradigmas en la concepción y desarrollo de ciencia, tecnología y producción de conocimiento, que deberían ser asumidas por las universidades de la región? Y si el cristal con que se mire fuera ciencia-tecnología-conocimiento, entonces ¿cómo nos vemos a través de él como universidad peruana frente a los escenarios universitarios de otros países?

Responder a estas interrogantes nos llevará por caminos tal vez transitados pero no asimilados a plenitud, dado que las políticas de desarrollo en ciencia y tecnología no están articuladas al quehacer universitario y empresarial, tal como lo exigen las actuales tendencias del desarrollo social que privilegian el capital del conocimiento. Entendiendo que ciencia y tecnología son herramientas indispensables en la construcción de sociedades modernas e incluyentes, se hace necesaria fortalecer la investigación científica y la innovación tecnológica como tareas imprescindibles para apoyar el desarrollo de las sociedades y para competir en un entorno cada vez más dominado por el conocimiento y la información; escenario en el cual la universidad asume una alta responsabilidad social.

\section{CONCLUSIONES}

1. El concepto de universidad tradicional ha cambiado en esta sociedad del conocimiento y la globalización. 
2. La falta de apoyo presupuestal y de políticas erradas hacia el sistema universitario por parte del Estado están sumergiendo a la universidad en una crisis coyuntural y estructural. Es necesaria la realización de una reforma integral de la educación superior, que asegure la calidad de la formación académica, donde la investigación es tarea prioritaria.

3. Se crearon universidades públicas por réditos políticos, sin presupuesto propio y sin evaluar la calidad de la educación, sin criterio educacional ni de desarrollo.

4. El desarrollo de la ciencia y la innovación debe ser promovida mediante la relación entre empresa privada, gobierno y universidades. En el ámbito de la investigación podríamos decir que el debate está en cómo desconcentrar los recursos de gestión y el uso de rentas para alcanzar una gestión de innovación,acortando la fisura digital para una mejor eficiencia.

5. La organización de las universidades requiere de cambio y desarrollo para una gestión administrativa transparente, eficiente y eficaz con participación democrática para una gestión que se base en una dirección estratégica con innovación y creatividad, en la búsqueda de una mejor formación profesional de excelencia y una concertación para el desarrollo.

6. En la actualidad, podemos afirmar que las universidades peruanas tienen dos retos fundamentales a enfrentar: la acreditación de la calidad educativa y el seguimiento homogéneo en su evaluación como una forma de generar una cultura de la calidad y del modelo educativo universitario.

7. Las universidades que no cumplan con los estándares internacionales y no estén acreditadas no podrán emitir títulos a nombre de la nación, solo certificados a nombre de su institución;de esta manera se exigirá y se incentivará el mejoramiento de la calidad educativa en la formación científica que cada universidad debe tener.

8. Se tiene que promulgar una nueva ley universitaria que obligue y financie la investigación en ciencia y tecnología y que, a su vez, desarrolle post grados, ya que en la actualidad están diseñadas para ofrecer estudios de pregrado y son pocas las que dirigen sus recursos para desarrollar programas de especialización con nuevos conocimientos.
9. En esta aldea global, la generación de ciencia y tecnología es un poder, para esto se requiere continuidad en la calidad en la formación y se necesita de herramientas como la predictibilidad, competencia y eficiencia delas universidades públicas. En ese sentido, podemos afirmar -en términos generales- que la educación y la innovación son el pasaporte para el futuro.

\section{BIBLIOGRAFÍA}

Aguirre Yato, Víctor y otros. (2007). Estrategias para el desarrollo de las Universidades del Perú. Lima, Asamblea Nacional de Rectores. Comisión Especial, designada por Resolución N³68-2007-ANR.

Asamblea Nacional de Rectores. Comisión Nacional de Rectores para la Acreditación, Dirección general de Investigaciones y acreditación Universitaria. (2004). Estándares Mínimos para la Autoevaluación de las carreras profesionales. Documento de Trabajo. Lima, Edit. ANR.

Brunner, J.J. (1998). Globalización Cultural y Postmodernidad, Breviarios. Santiago de Chile, Fondo de Cultura Económica.

Carnoy, Martín. (1999). Globalization and educationalreform: whatplannersneedtoknow. París, IIP - UNESCO.

Carvallo, Constantino. (2009). Diario Educar. Lima, Editorial Aguilar.

Cisneros, Jaime Luis. (2009). Aula Abierta. Lima, Editorial Norma; Penac.

Ciudadanos al Día (CAD). Crecimiento de las universidades privadas. Información publicada por la ANR.

Consorcio de Universidades. (1999). Autoevaluación institucional: Manual para Instituciones de educación superior. Lima, Fondo Editorial Universidad del Pacífico.

De Paz Toledo, Zenón y Cuba Sancho, Juana. (2003). Lineamientos Para una política de calidad, autoevaluación y acreditación. Lima, Editorial UNMSM.

Delgado de la Flor Badaraco, Francisco y Alcázar C., Marcia. (2004). "Informe sobre Educación Superior Universitaria”. Lima, Asamblea Nacional de Rectores. Ver: Aguirre Yato, Víctor y otros. (2008). Estrategias para el 
desarrollo de las Universidades del Perú. Lima, Asamblea Nacional de Rectores.

Gibbons, Michael. (1997). The new production of Knowledge (La nuevaproducción del conocimiento). The dynamic of science and research in contemporary societies.LondonNew Delhi, ThousandOaks.

Gómez Buendía, Hernando (1998). PNUD: Educación. La agenda del siglo XXI. Hacia un desarrollo humano. Bogotá, TM Editores.

Gutiérrez Huby, Ana María. (2006). "Formulación y desarrollo de un modelo de autoevaluaciónpara la acreditación del Programa de Contabilidad". Tesis para optar el Grado Académico de Magíster en Administración, mención Gestión Empresarial. Lima, UNMSM.

Licha, Isabel. (1996). La investigación y las universidades latinoamericanas en el umbral delsiglo XXI: Los desafíos de la globalización. Colección UDUAL 7. México D.F., UDUAL,

López Más, Julio; Cabrejos Polo, Jorge y Gutiérrez Huby, Ana María. (2006). Guía de Autoevaluación para la acreditación en el área económico empresarial. Lima, Editorial UNMSM.

LopezSegrera, Francisco. (2001). Globalización y Educación Superior en América Latina y el Caribe. Caracas, Ediciones IESALC/UNESCO.

Marticorena Castillo, Benjamín. (2007). Ciencia, tecnología y sociedad en el Perú: Memoria CONCYTEC 2001-2006. Lima, CONCYTEC.

Ministerio de Educación. (2004). En Torno al sentido de la educación superior universitaria. Cuadernos de reflexión y debate. Lima, Oficina de Coordinación Universitaria.

Mondolfo, Rodolfo. (1966). Universidad: Pasado y Presente. Buenos Aires, Editorial Universitaria de Buenos Aires.

Ongaro Estrada, Andrés A. (2006). La Legislación sobre Educación Superior en el Perú. Lima, Edit. ANR.

Rama Vitale, Claudio. (2009). Tendencias de la educación Superior en América Latina y El Caribe en el Siglo XXI. Tomo II. Lima, Asamblea Nacional de Rectores.

Robbins, Stephen P. (1996). "Cultura organizacional”. En Comportamiento Organizacional.
México, Prentice Hall Hispanoamericana.

Rodríguez Gómez, Roberto. (2006). "El Porvenir de la Universidad Latinoamericana". En: Revista CRESAL / UNESCO. N³/06.Caracas, editorial Ciane.

Rojas Mix, Miguel. (2007). El Compromiso social de las Universidades de América Latina y el Caribe. Declaración del Congreso Internacional de Rectores Latinoamericanos y Caribeños. Bello Horizonte, Brasil.

Stiglitz, Joseph E. (2002). El malestar en la globalización. Madrid, Taurus-Santillana.

Tapia, Marta, Presidenta del Consejo Nacional para la Autorización de Universidades (CONAFU). (2012). Informe. Lima, CAD.

UNESCO. (1998). La Ciencia para el Siglo XXI: Una nueva visión y un marco para la acción. Montevideo, UNESCO.

Universidad de Oviedo, Plan Nacional de Evaluación de la Calidad de las Universidades. Consejo de las universidades. (1999). Informe del Comité Técnico para las universidades participantes. Convocatoria diciembre 1999. Oviedo-España, Monografía.

Universidad Nacional Mayor de San Marcos. Consejo de Transferencia e Innovación, Vicerrectorado de Investigación. (2007). I Feria de Transferencia e Innovación Tecnológica, 19,20 y 21 de Julio 2007. Lima, Editorial UNMSM.

Universidad Nacional Mayor de San Marcos. (2006). Lineamientos para una Política de Calidad, Autoevaluación y Acreditación en la UNMSM. Lima, Documento de Trabajo.

Universidad Nacional Mayor de San Marcos. (2007). Guía 2007 de Autoevaluación y Acreditación, pre grado y postgrado; UNMSM. Lima, Oficina central de Calidad y Acreditación de la UNMSM, Editorial UNMSM.

Universidad Nacional Mayor de San Marcos. Oficina Central de Calidad Académica y Acreditación de la UNMSM. (2005). Hacia la Autoevaluación en San Marcos. Lima, Editorial UNMSM.

Wong Torres, Zelma. (2004). "El Idioma Chino, lengua del futuro". En: Revista Quipukamayoc. Facultad de Ciencias Contable de la Universidad Nacional Mayor de San Marcos. Lima, Editorial UNMSM. 
Yarzábal, Luisa (2002): "La Educación Superior en la sociedad contemporánea”. Conferencia dictada en el "Primer Foro Nacional de la Educación Superior”, Managua, mayo de 2002. En: Universidad y Sociedad. Revista del Consejo Nacional de Universidades, $\mathrm{N}^{\circ} 10$, septiembre 2002, Managua.

\section{INTERNET}

Bunge, Mario (2007). "Conferencia Ciencia y Desarrollo en América Latina Hoy”. Desarrollada el 15 de noviembre del 2007. Canadá. En línea: http://nopiedra.wordpress. com/2007/11/15/ciencia-y-desarrollo-enamerica-latina-hoy-mario-bunge/. Consulta hecha el 22 de noviembre del 2007.

Bunge, Mario. (1996) La Ciencia su Método y su Filosofía. Extraído de la página [http://www.monografias.com/trabajos16/ ciencia-y-tecnologia/ciencia-y-tecnologia. shtml\#CONCEPT. Consulta hecha el 17 de noviembre del 2007.

Chen, Susan. (2004). En: Revista de las Sedes Regionales de Costa Rica. Intercedes. Vol. V. $\mathrm{N}^{\circ} 7$. Costa Rica. En http://www.intersedes. ucr.ac.cr/07-autores.html\# autor06. Consulta hecha el 21 de noviembre del 2007.

Galán, Adelaida y otros. (2006). Crónica del Primer Congreso Iberoamericano de Ciencia, Tecnología, Sociedad e Innovación “Ciencia, Tecnología e Innovación para el desarrollo en Iberoamérica”. México D.F. 19 al 23 de junio del 2006. En: http://www.revistacts. net/3/7/09/file.Extraído el 18 de noviembredel 2007.

World Bank Group. (2004). "Key Development Data \& Statistics 2004".Citado en la revista:Conduct in anEmergingMarketEconomy.Documento en línea: http:// www.ita.doc.gov/goodgovernance/adobe/ Bus_Ethics_sp/Chapter\%20I/I_Chapter_1. pdf. Consulta el 19 de noviembre del 2011.

www.concytec.gob.pe.

www.iesalc.unesco.org.ve/.../Art\%EDculo\%20-\%20 Etica\%20y\%20educaci\%F3n\%20superior.pdf

www.wikipedia.org/wiki/universidad

\section{NORMAS LEGALES}

Constitución Política del Perú 1993.

Ley $\mathrm{N}^{\circ} 28740$, promulgada el 19 de Mayo del 2006 y publicado su Reglamento el 10 de julio del 2007.

Ley $\mathrm{N}^{\circ} 28044$, Ley General de Educación.

Ley $\mathrm{N}^{\circ} 27806$, Ley de Transparencia y Acceso a la Información.

Ley $\mathrm{N}^{\circ} 28303$ establecida en el Decreto Supremo $\mathrm{N}^{\circ} 082-2005-\mathrm{PCM}$, referente a su adscripción al Ministerio de Educación y la Ley del CONCYTEC. Ver Portal del CONCYTEC. 